\title{
A brief overview of loop quantum cosmology
}

\author{
Julien Grain** \\ CNRS, Institut d'Astrophysique Spatiale, UMR8617,Orsay, France, F-91405 \\ and \\ Université Paris Sud 11, Orsay, France, F-91405 \\ E-mail: julien.graineias.u-psud.fr
}

Loop quantum cosmology (a symmetry-reduced quantum model of the Universe inspired by loop quantum gravity) extends the inflationary paradigm to the Planck era: the big bang singularity is replaced by a quantum bounce naturally followed by inflation. Testing for these models requires to compute the amount of cosmological perturbations produced in this quantum background and subsequently derives their footprints on the cosmic microwave background. This proceedings proposes a very brief summary of this road from quantum gravity to cosmological observables, focusing on the case of a loop-quantum model of flat FLRW spaces with cosmological perturbations. The background evolution, passing through a bounce, is first presented. The case of cosmic inhomogeneities in a perturbative treatment about a quantum background are then discussed (with an emphasis on two specific theoretical constructions), and there respective predictions in terms of primordial power spectra for the tensor modes are outlined.

Frontiers of Fundamental Physics 14 - FFP14,

15-18 July 2014

Aix Marseille University (AMU) Saint-Charles Campus, Marseille

\footnotetext{
* Speaker.

$\dagger$ Summary of researches mainly done in collaboration with A. Barrau, T. Cailleteau, and J. Mielczarek. Many thanks to J. Ben Achour, M. Bojowald, B. Bolliet, G. Calcagni, A. Ferté, M. Kagan, L. Linsefors, E. Livine, K. Noui, L. Smolin, C. Stahl, F. Vidotto and E. Wilson-Ewing for useful discussions and/or works altogether.
} 
Introduction- Loop quantum cosmology (LQC) [1] is a tentative approach to built a quantum model of the Universe inspired by the ideas of loop quantum gravity (LQG) -a non-perturbative quantization of general relativity- [2], thus allowing for extending the inflationary paradigm to the Planck era where quantum gravity effects are no more negligible. This approach relies on minisuperspace ideas: the entire phase space of general relativity is reduced thanks to the isotropy and homogeneity symmetries, meaning that only FLRW metric are considered. This reduced phase space is then quantized using the loop representation. At a quantum level, the big bang singularity issue is solved for the transition to the null volume is forbidden, leading to the replacement of the big bang singularity by a quantum big bounce. This is understood at a more effective level by a regularization of the curvature. Classically, the curvature can be computed from holonomies evaluated around a plaquette and then, by shrinking the area of the plaquette to zero. (The FLRW space can be paved by identical plaquettes thanks to isotropy and homogeneity.) However, the kinematical Hilbert space in LQG is given by eigenstates of geometric (area and volume) operators which have discrete spectra. This leads to a minimal area gap and the area of the plaquette around which holonomies are evaluated cannot be shrinked to zero anymore, thus preventing the curvature to diverge. This is captured in the effective, modified Friedmann equation (for a flat FLRW metric with holonomy correction only): $\left(\frac{\dot{a}}{a}\right)^{2}=\frac{8 \pi G}{3} \rho\left(1-\frac{\rho}{\rho_{c}}\right)$, with $\rho_{c}$ a maximal energy density precisely set by the minimal area gap ( $\rho_{c}$ is of the order of the Planck energy density, though its explicit value depends on the value of the Barbero-Immirzi parameter, $\gamma$ ). In the effective picture, the bounce occurs when the Hubble parameter, $H:=(\dot{a} / a)$, vanishes, that is when the energy density of the content of the universe reaches $\rho_{c}$. The classical equation is recovered for $\rho \ll \rho_{c}$. The cosmic history would therefore be a phase of classical contraction ( $\rho \ll \rho_{c}$ and $\rho$ increases), then a quantum bounce $\left(\rho \lesssim \rho_{c}\right.$ ) making contact with a classical expansion ( $\rho \ll \rho_{c}$ and $\rho$ decreases). (The efficiency of this approach has been shown thanks to both numerical and analytical arguments [3].)

Obviously, LQC is not free of any hypotheses (though following the spirit of LQG, it attempts to make use of few of them), and its program is not fully accomplished yet. The robustness of any bouncing cosmologies should be tested against the evolution of anisotropies through the bounce, and now a rather wide amount of studies is devoted to this question (see [4] and references therein). Similarly, its robustness against of full derivation of the quantum cosmological history starting from LQG is still needed, and recent progresses have been made in e.g. spin foams or group field theory [5]. In any case, the last years of research in this field have paved a road going from quantum gravity to cosmological observables. Here we focus on a paradigmatic (and most advanced) example of such a program. We consider the case of LQC for flat FLRW spaces incorporating cosmological perturbations. The matter content is a massive scalar field allowing for having a phase of inflation shortly after the bounce. Finally, only holonomy corrections are considered here (see e.g. [6] for inverse volume corrections).

Background- The effective equation of motions for the background are obtained by regularizing the curvature operator. The gravitational phase space is composed of the extrinsic curvature, $k$, and $p:=a^{2}$ while the phase space for matter is the field configuration, $\varphi$, and its conjugate momentum, $\Pi$. Their associated Poisson brackets are $\{k, p\}=\frac{8 \pi G \gamma}{3}$ and $\{\varphi, \Pi\}=1$. The effective Hamiltonian is derived by replacing $k \rightarrow \frac{\sin (\mu \gamma k)}{\mu \gamma}$, with $\mu \gamma:=\sqrt{\Delta / p}$ and $\Delta=2 \pi \gamma \sqrt{3} L_{\mathrm{Pl}}^{2}$ is fixed by the minimal area gap of LQG ( $L_{\mathrm{Pl}}$ is the Planck length). This yields the effective Hamiltonian $(V(\varphi)$ 
is the massive potential of the scalar field): $\mathscr{H}^{\text {eff }}=-\frac{3 \sqrt{p}}{8 \pi G \gamma^{2}}\left(\frac{\sin (\mu \gamma k)}{\mu \gamma}\right)^{2}+\left(\frac{\Pi^{2}}{2 p^{3 / 2}}+p^{3 / 2} V(\varphi)\right)$. From the Hamilton equations and the fact that such an Hamiltonian is a constraint ( $\mathscr{H}^{\mathrm{eff}} \approx 0$ on physical solutions), one easily derived the effective Friedmann equation given previously, with $\rho=(\dot{\varphi})^{2} / 2+V(\varphi)$. Similarly, the Klein-Gordon equation governing the evolution of the scalar field is $\ddot{\varphi}+3 H \dot{\varphi}+\partial_{\varphi} V(\varphi)=0$. The solutions of the above set of equations obviously go through a bounce for $H=0$ (with $H<0$ during the classical contraction and $H>0$ during the classical expansion), corresponding to $\rho=\rho_{c}{ }^{1}$.

Though LQC is not a complete deduction of LQG, this class of models shows interesting features. The first of them is, obviously, the fact that the big bang singularity is systematically removed (and there are good hints that this is a general feature of LQG [7]). Second, this approach has been extended to non-flat FLRW metrics as well as to anisotropic models such as Bianchi models, to show that the bounce remains [4] (note that the presence of the bounce is robust against different sort of classical content of the universe since it is here a purely quantum gravitational feature). Finally, if one consider our Universe to be filled by a massive scalar field during its primordial ages (as modelled in inflation), it has been shown that most of the solutions of the effective equation go through a phase of inflation shortly after the quantum bounce with the sufficient amount of $\sim 60$ e-folds [8]. Though this a priori depends on when the initial conditions are chosen and what is the natural PDF for them, this conclusion seems rather robust in the context of flat FLRW spaces (see also [4] for the case of anisotropic models). The fact that the universe experienced a phase of cosmic inflation shortly after the bounce is intuitively understood as follows. During the contraction, the scalar field oscillates in its massive potential with a rough frequency given by its mass. However $H$ is negative-valued and then acts as antifriction during the contraction (through the term $3 H \dot{\varphi}$ ). This amplifies the amplitude of oscillations up to a point (very close to the bounce) where the amplification is dominating the dynamics of $\varphi$. This pushes up the field very high in its potential during the bounce and, as a consequence, the scalar field is very high in its potential while the Universe has just entered in its expanding phase. This is precisely the appropriate conditions for a phase of slow-roll inflation to start.

Inhomogeneities- These models have been extended to include the case of cosmological perturbations, which are our major observationnal probe of the physics of the Universe across cosmic times. This offers the possibility to compute the amount of cosmological inhomogeneities produced in the primordial Universe and how the peculiar cosmic history, passing through a bounce, affects them. These possible impact can then be turned into an impact on cosmological probes such as the cosmic microwave background (CMB) anisotropies, allowing for bridging contact with observations. These extension are not a priori solely given by the impact of a bounce on the standard cosmological perturbations for this would not take into account the peculiar fact that these inhomogeneities propagate on a quantum background. Indeed, standard cosmological perturbations are derived by perturbing the Einstein's equations about a classical background, and, in this case, one

\footnotetext{
${ }^{1}$ In the effective approach, this scale factor is viewed classically but its evolution is dictated by a modified Friedmann equation. In a quantum setting, one is interested in the evolution of a wave function for the Universe. Sharply peaked states (standing for wave functions peaked around the classical solutions in the large volume limit) are selected. During its evolution, this state remains peaked and the scale factor solution of the modified Friedmann equation can be interpreted as the value of the scale factor which is traced by the peak of the state.
} 
has to consider that our starting point is neither a classical background, nor the Einstein's equations. Different approaches have been developped $[9,10,11]$ and we will focus on two of them: the deformed algebra approach [10] and the dressed metric approach [9].

Cosmological perturbations are obtained by extending the phase space to include inhomogeneous degrees of freedom in a perturbative setting: the different constraints of general relativity (the scalar, diffeomorphism and Gauss constraints) are expanded up to second order in perturbations about the FLRW background. The zeroth order leads to the background equations and the second order generates the dynamics of the perturbations in this background. Finally, the first order perturbation of the constraints generates the gauge transformations for perturbations: quotiented the perturbative part of the phase space by these transformations allows for defining the gauge invariant (GI), physically relevant, perturbative degrees of freedom.

In a sense, the deformed algebra approach consists in perturbing the effective, background space [12], ensuring the perturbed theory to remain anomaly-free. Technically, the constraints expanded up to the second order are phenomenologically corrected to account for the background quantum corrections. These modifications are then univoquely determined (at least for holonomy corrections [13]) requiring that the classical equations are recovered in the large volume limit, and, that the constraints up to second order do form an algebra and thus remains first class, on-shell and off-shell (as is the case in the classical theory). This univoquely defined the second order hamiltonian (generating the dynamics) and the first order one (generating the gauge transformations) both modified at an effective level. (We note that for a matter content given by a single scalar field, the GI perturbations are still composed of two tensor modes, corresponding to the two helicity states of gravitational waves, and one scalar mode.) As an example, the equation of motion for the tensor modes ( $v_{k}$ is the Mukhanov-Saski variables of wavenumber $k$ ) reads $v_{k}^{\prime \prime}+\left(\Omega k^{2}-z^{\prime \prime} / z\right) v_{k}=0$, with a prime denoting differentiation with respect to conformal time, $d \eta=d t / a(t), \Omega=1-2 \rho / \rho_{c}$ and $z=a / \sqrt{\Omega}$. It appears that this procedure can be captured by a simple modification of the structure functions of the algebra of constraints, as compared to the classical case, which could potentially lead to a change of signature at the effective level for $\rho_{c} / 2 \leq \rho \leq \rho_{c}$ [10]. Finally, the GI perturbative degrees of freedom are quantized using Fock quantization on curved spaces.

The dressed metric approach adopts a different path, and in a sense, it is a pure minisuperspace approach with perturbations. The perturbative part of the extended phase space is first quotiented by the gauge transformation generated by the classical first order constraints. (The GI degrees of freedom are thus obviously composed of two tensor modes and one scalar mode, as in the classical theory.) The background part of the phase space is then quantized $\grave{a}$ la loop while the GI perturbative degrees of freedom are quantized using techniques developped for the quantization of a test field in a LQC, quantum background [14]. The equation of motion for the tensor modes functions is finally given by an equation of motion similar in its form to the classical theory, but introducing a metric which is dressed up by quantum corrections (with potential ambiguities in defining it, see [14]). For the tensor mode, this reads $v_{k}^{\prime \prime}+\left(k^{2}-\tilde{a}^{\prime \prime} / \tilde{a}\right) v_{k}=0$ with $\tilde{a}^{4}=\left\langle\hat{H}_{b c k g}^{-1 / 2} \hat{a}^{4} \hat{H}_{b c k g}^{-1 / 2}\right\rangle /\left\langle\hat{H}_{b c k g}^{-1}\right\rangle$ the dressed scale factor and $\langle\cdot\rangle$ the quantum expectation value on the background state.

Primordial power spectra- The equation of motion for perturbations are the starting point for computing the primordial power spectra of cosmic inhomogeneities evolving through (and amplified by) the classical contraction, the quantum bounce and the cosmic inflation. For tensor modes, 


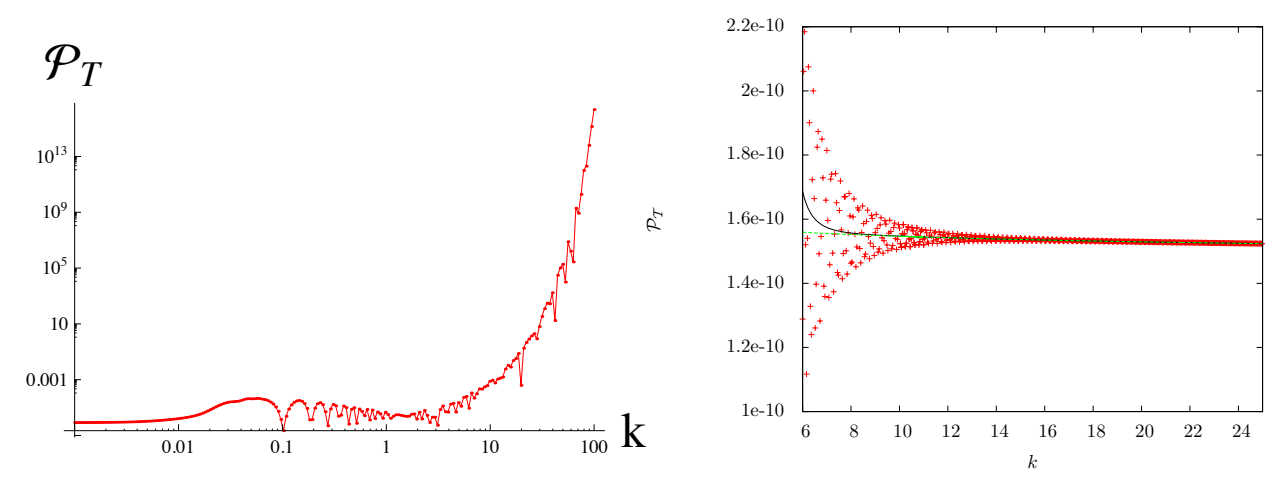

Figure 1: Primordial power spectrum for tensor modes as derived in the deformed algebra approach (left, from [15]), and in the dressed metric approach (right, from [9]).

it is defined as $\mathscr{P}_{T}(k)=\left(32 G k^{3} / \pi\right)\left|v_{k}\left(\eta_{f}\right) / a\left(\eta_{f}\right)\right|^{2}$, with $\eta_{f}$ denoting the end of inflation (the sum over the two helicity states is implicitly done here). We remind that in the pure inflationary paradigm, the predicted primordial power spectra are given (at first order in slow-roll) by a power law with a very slightly negative spectral index.

In the case of the deformed algebra approach, the equation of motion becomes elliptic for $\rho_{c} / 2 \leq \rho \leq \rho_{c}$ : it appears more natural to set the initial conditions in the contracting phase $(\rho \ll$ $\rho_{c} / 2$ ) for which a Minkowki vacuum is easily defined (the equation of motion is then hyperbolic allowing for solving an initial value problem). The derived power spectrum essentially shows two regimes [15] (Fig. 1, left). For $k<\sqrt{8 \pi G \rho_{c}}, \mathscr{P}_{T}(k)$ is scale invariant in the infrared and then shows some oscillations. This corresponds to modes which are amplified by the effective space curvature (the term $z^{\prime \prime} / z$ ) during the contraction and the bounce. For $k>\sqrt{8 \pi G \rho_{c}}, \mathscr{P}_{T}(k)$ increases exponentially. This comes from the change of signature which manifests as an instability (the term $\Omega k^{2}$ is negative valued around the bounce) if one sets the initial conditions in the remote past. (Note that one could alternatively solved a boundary value problem fixing the boundary in the elliptic regime.) In the dressed metric case, initial conditions can be set either at the bounce or in the remote past. An example of the spectrum chosing a 4th order WKB vacuum at the bounce is shown on the right of Fig. 1 [9]. For $k>\sqrt{8 \pi G \rho_{c}}$, the standard inflationary spectrum is recovered. This corresponds to $k$-modes which feel the space curvature during inflation only. For $k<\sqrt{8 \pi G \rho_{c}}$, the spectrum reddens as these modes are sensitive to the space curvature during the bounce. A similar shape is obtained for the scalar mode, meaning that the impact of LQC in this scheme translate into a positive running of the spectral index.

Conclusion- Those primordial power spectra of cosmic inhomogeneities are the source of the CMB anisotropies. There impact on the angular power spectra of the CMB anisotropies is easily computed from numerical solutions of the Boltzmann and radiative transfer equations. Since LQC-spectra differs from the inflationary prediction, the specific features imprinted by the quantum bounce on the cosmic inhomogeneities could in principle be searched for in the CMB data. A quantitative study and comparison with datasets, as well as a wider exploration of the initial conditions and the potential features of the quantum bounce, are however still to be done. 


\section{References}

[1] M. Bojowald, Quantum Cosmology, Springer, New-York (2011); K. Banerjee, G. Calcagni, and M. Martin-Benito, SIGMA 8016 (2012); M. Bojowald, Living Rev. Rel. 11, 4 (2008); A. Ashtekar, M. Bojowald, \& J. Lewandowski, Adv. Theor. Math. Phys. 7, 233 (2003) A. Barrau, T. Cailleteau, J. Grain, J. Mielczarek, Class. Quantum Grav. 335, 053001 (2014);

[2] C. Rovelli \& F. Vidotto, Covariant Loop Quantum Gravity, Cambridge University Press (2014); R. Gambini \& J. Pullin, A First Course in Loop Quantum Gravity, Oxford, Oxford University Press (2011); T. Thiemann, Modern Canonical Quantum General Relativity, Cambridge, Cambridge University Press (2007); C. Rovelli, Quantum Gravity, Cambridge, Cambridge University Press (2004);

[3] A. Ashtekar, T. Pawlowski \& P. Singh, Phys. Rev. D 73124038 (2006); A. Ashtekar \& P. Singh, Class. Quant. Grav. 28213001 (2011); C. Rovelli \& E. Wilson-Ewing, Phys. Rev. D 90023538 (2014)

[4] L. Linsefors \& A. Barrau, Class. Quant. Grav. 31015018 (2014) and arXiv:1405.1753[gr-qc]

[5] C. Rovelli \& F. Vidotto, Class. Quant. Grav. 25225024 (2008) and Class. Quant. grav. 27145005 (2010); E. Bianchi, C. Rovelli \& F. Vidotto, Phys. Rev. D 82084035 (2010); A. Henderson, C. Rovelli, F. Vidotto \& E. Wilson-Ewing, Class. Quant. Grav. 28025003 (2011); S. Gielen, D. Oriti \& L. Sindoni, Phys. Rev. Lett. 111031301 (2013) and arXiv :1311.1238[gr-qc]; G. Calcagni, S. Gielen \& D. Oriti, Class. Quant. Grav. 29105005 (2012)

[6] J. Grain, T. Cailleteau, A. Barrau \& A. Gorecki, Phys. Rev. D 81024040 (2010); M. Bojowald, G. Calcagni \& S. Tsujikawa, JCAP 11046 (2011) and Phys. Rev. Lett. 107211302 (2011)

[7] C. Rovelli \& F. Vidotto, Phys. Rev. Lett. 111091303 (2013); P. Singh \& F. Vidotto, Phys. Rev. D 83 064027 (2011)

[8] A. Ashtekar \& D. Sloan, Phys. Lett. B 694108 (2012); A. Ashtekar \& D. Sloan, Gen. Rel. Grav. 43 3519 (2011); L. Linsefors \& A. Barrau, Phys. Rev. D 87123509 (2013)

[9] I. Agullo, A. Ashtekar \& W. Nelson, Phys. Rev. Lett. 109251301 (2012); I. Agullo, A. Ashtekar \& W. Nelson, Phys. Rev. D 87043507 (2012); I. Agullo, A. Ashtekar \& W. Nelson, Class. Quantum Grav. 30085014 (2013);

[10] J. Mielczarek, T. Cailleteau, A. Barrau \& J. Grain, Class. Quant. Grav. 29085009 (2012); T. Cailleteau, J. Mielczarek, A. Barrau \& J. Grain, Class. Quant. Grav. 29095010 (2012); T. Cailleteau, A. Barrau, J. Grain \& F. Vidotto, Phys. Rev. D 86087301 (2012); A. Barrau, M. Bojowald, J. Grain \& M. Kagan, arXiv:1404.1018[gr-qc]; M. Bojowald \& G. M. Paily, Phys. Rev. D 86104018 (2012)

[11] G. A. Mena Marugan \& M. Martin-Benito, Int. J. Mod. Phys. A24 2820 (2009); L. J. Garay, M. Martin-Benito \& G. A. Mena Marugan, Phys. Rev D 82044048 (2010); M. Fernandez-Mendez, G. A. Mena Marugan \& J. Olmedo, Phys. Rev. D 86024003 (2012)

[12] E. Wilson-Ewing, Class. Quant. Grav. 29085005 (2012)

[13] M. Bojowald, G. M Hossain, M. Kagan \& S. Shankaranarayanan, Phys. Rev. D 78063547 (2008) and Phys. Rev. D 79043505 (2009) [Erratum-ibid. D, 82, 109903, 2010]; M. Bojowald \& G. M. Hossain, Class. Quant. Grav. 244801 (2007) and Phys. Rev. D 77023508 (2008); T. Cailleteau, L. Linsefors \& A. Barrau, Class. Quant. Grav. 31125011 (2014)

[14] A. Ashtekar, W. Kaminski \& J. Lewandowski, Phys. Rev. D 064030 (2009), A. Dapor \& J. Lewandowski, Phys. Rev. D 87063512 (2013)

[15] L. Linsefors, T. Cailleteau, A. Barrau \& J. Grain, Phys. Rev. D 87107503 (2013) 